\title{
Surface characterization of polymers used in fabrication of interim prostheses after treatment with photopolymerized glaze
}

\author{
Daniela Micheline dos Santos ${ }^{\mathrm{a}, *}$, Betina Chiarelo Commar ${ }^{\mathrm{a}}$, Liliane da Rocha Bonatto ${ }^{\mathrm{a}}$, \\ Emily Vivianne Freitas da Silva a ${ }^{a}$, Mariana Vilela Sônego ${ }^{a}$, Elidiane Cipriano Rangel ${ }^{\text {b }}$, \\ Aldieris Alves Pesqueira ${ }^{a}$, Marcelo Coelho Goiato ${ }^{a}$ \\ a Department of Dental Materials and Prosthodontics, Aracatuba Dental School, Univ Estadual Paulista (UNESP), José Bonifácio St., 1193, Aracatuba, São Paulo 16015-050, Brazil \\ b Technological Plasma Laboratory (LaPTec), Experimental Campus of Sorocaba, Univ Estadual Paulista (UNESP), Tres de Março Av , 511, Sorocaba, Sao Paulo, 18087-180, Brazil
}

\section{A R T I C L E I N F O}

Article history:

Received 3 October 2016

Accepted 23 October 2016

Available online 26 October 2016

\section{Keywords:}

Composite resins

Acrylic resins

Color

Hardness

Chemical polishing

\begin{abstract}
A B S T R A C T
The material used for interim prostheses fabrication must present excellent physical properties for greater longevity in the face of environmental conditions, which can occur in the oral cavity. The purpose of this study was to evaluate the effect of a photopolymerized glaze on the physical and mechanical properties of polymers used for the fabrication of interim prostheses, before and after thermocycling and immersion in staining solutions. One hundred samples of composite and acrylic resins were fabricated: Dencor chemically activated acrylic resin (CAAR) $(n=20)$ and heat-polymerized acrylic resin (HPAR) $(n=20)$, Charisma $(n=20)$, Structur $(n=$ $20)$, and Protemp $(n=20)$. A mechanical polishing was performed on half of the samples, and a chemical polishing was performed on the remaining samples. Subsequently, all samples were submitted to thermocycling and immersion in coffee staining solution for 21 days. Analysis of color and microhardness, as well as atomic force microscopy (AFM), scanning electron microscopy (SEM), and energy dispersive $x$-ray spectrometry (EDS) were performed. The data were submitted to repeated-measures analysis of variance (ANOVA), followed by the Tukey test $(\alpha=0.05)$ and the Student $t$-test $(\alpha=0.05)$. It was verified that the glaze decreased the chromatic alteration values, and increased the microhardness values of the samples, with the exception of the Charisma resin. The samples that did not receive chemical polishing had the greatest number of surface irregularities. This study concluded that the groups with glaze presented less color alteration. In addition, Charisma and Structur resins exhibited the greatest chromatic stability. As to the microhardness, the values were greater when the samples were treated with the glaze, with the exception of the Charisma group.
\end{abstract}

(C) 2016 Elsevier B.V. All rights reserved.

\section{Introduction}

Adequate interim restorations must furnish the esthetic, functional, and biologic needs of the patient [1-4]. In some cases such as periodontal surgeries, rehabilitations involving implant installations, or maxillofacial rehabilitations [3], the use of interim restorations can be indicated for a greater period $[3,5]$. However, the physical and mechanical properties of the materials could suffer alterations over time, due to the action of the adverse environment of the oral cavity where the sorption of water and other liquids occur, decreasing the longevity of the restoration [6]. Therefore, the material used must present excellent physical and mechanical properties to achieve greater longevity $[7,8]$.

\footnotetext{
* Corresponding author at: Department of Dental Materials and Prosthodontics, Aracatuba Dental School, Univ. Estadual Paulista, UNESP, Jose Bonifacio St., 1153, Vila Mendonca, Aracatuba, Sao Paulo, Brazil, 16015-050.

E-mail address: danielamicheline@foa.unesp.br (D.M. Santos).
}

Chromatic stability is the property a material possesses to retain its color for a period of time in a determined environment, which is an important physical property for dental materials $[9,10]$. The alteration of color in polymeric materials can be caused by intrinsic and extrinsic factors [10-14]. Modifications in the polymeric matrix with chromatic alteration of the restorative material are caused by intrinsic factors [10, 13]. Extrinsic factors such as solar radiation, thermal changes, humidity, absorption, and adsorption of substances can also cause discoloration [10-14]. Also, the surface and luminosity of the object must be considered as influencing factors in the determination of the color [15].

Another characteristic which can be influenced by the oral cavity is the surface microhardness, characterized by the resistance of the material to a permanent penetration. (artigo bruna) This characteristic is related to other properties of the material, such as the wear resistance [16-18]. Restorations suffer physical and mechanical alterations over time, causing surface degradation which allows the formation of microfractures that interfere in the maintenance of treatment [17-19]. 
Many materials and techniques exist for mechanical polishing of polymers to increase the surface smoothness, and therefore decrease porosity and bacterial adhesion. These include the use of abrasive bits with different granulations, and/or chemical polishing with the use of chemical substances on the material $[20,21]$. Currently, liquid polishing with photopolymerized sealants is being used to reduce the stages of polishing, providing a smooth and polished surface, and avoiding the accumulation of biofilm [22].

Therefore, the purpose of this study was to evaluate the influence of the application of photopolymerized glaze on the physical and mechanical properties of polymers used in the fabrication of interim prostheses, before and after thermocycling and immersion in coffee staining solution. The null hypothesis is that the application of photopolymerized glaze, and the processes of thermocycling and immersion of the samples, does not influence the physical and mechanical properties of the polymers used in the fabrication of interim prostheses.

\section{Materials and methods}

\subsection{Sample preparation}

Five different types of resins ( $n=20)$ were analyzed: resin composed of Bis-acryl from Protemp (3 M/ESPE, Seefeld, Germany) and Structur (VOCO, Vuxhaven, Germany); resin composed of Bis-GMA from Charisma (Heraeus Kulze South America Ltda, Sao Paulo, Sao Paulo, Brazil); and autopolymerized (CAAR) and heat-activated (HPAR) acrylic resins, from Dencor (Artigos Odontologicos Classico Ltda, Sao Paulo, Sao Paulo, Brazil).

A $3 \mathrm{~mm}$ thick molded metal matrix was used for standardization of the samples. The matrix contained 10 circular compartments in its interior, each with a $10 \mathrm{~mm}$ diameter [23].

The specimens were divided into 10 groups $(n=10)$ : Protemp without photopolymerized glaze (A), Protemp with photopolymerized glaze (B), Charisma without photopolymerized glaze (C), Charisma with photopolymerized glaze (D), CAAR without photopolymerized glaze (E), CAAR with photopolymerized glaze (F), HPAR without photopolymerized glaze $(G)$, HPAR with photopolymerized glaze $(H)$, Structur without photopolymerized glaze (I), Structur with photopolymerized glaze (J).

During the fabrication of the samples, the inferior portion of the matrix was supported over a glass slide, a thin layer of solid petroleum jelly was applied, and the entire cavity was filled with the CAAR or composite resins (Charisma, Structur, or Protemp). Another glass slide was positioned over the resin layer to drain the excess material, maintaining the surface smooth and homogeneous. Subsequently, the samples were polymerized according to the manufacturer's recommendations [24,25].

For the fabrication of the HPAR samples, the metal matrix and glass slide set was placed in a special muffle for microwave ovens (Artigos Odontologicos Classico Ltda, Sao Paulo, Sao Paulo, Brazil) with special plaster type IV (Durone; Dentsply Ind and Com Ltda, Petropolis, Rio de Janeiro, Brazil) and a proportion of $100 \mathrm{~g}$ of powder to $30 \mathrm{~mL}$ of water, spatulated for $1 \mathrm{~min}$, and then poured under constant vibration. After the crystallization of the plaster, a second glass slide was positioned over the matrix already included in the plaster. A counter-muffle was positioned and special plaster type IV was poured over this last glass slide. Subsequently, the muffle was taken to the hydraulic bench press (VH Midas Dental Produtos Ltda, Araraquara, Sao Paulo, Brazil) and was put under a constant pressure of 1.25 tons for $3 \mathrm{~min}$. After the crystallization of the plaster, the muffle was opened and the glass surface was cleaned with acetone (Labsynth Produtos Laboratorios Ltda, Diadema, Sao Paulo, Brazil). The HPAR was proportioned according to the manufacturer's instructions. The resin was polymerized by microwave energy (Brastemp, Sao Paulo, Sao Paulo, Brazil) for 3 min with $30 \%$ power, then 4 min with 0\% power, and finally, 3 min with $60 \%$ power, according to the manufacturer's instructions [26,27]. After the polymerization, the samples were submitted to finishing with Maxicut bits (Vicking, Sao Paulo, Sao Paulo, Brazil) for excess removal [26].

Subsequently, a polishing of all samples was performed in an automatic polisher (Ecomet 300PRO; Buehler, Illinois, USA) with 400- and 800-grain metallographic sandpaper (Buehler, Illinois, USA), under constant water irrigation at a velocity of $300 \mathrm{rpm}$ [28]. Groups that did not receive the additional layer of photopolymerized glaze underwent additional polishing with 1000- and 1200-grain sandpaper, and the completion of the polishing with a diamond solution on a felt disc (Buehler, Illinois, USA). Each disc had its thickness measured with the assistance of a digital caliper (500-171-20B; Mitutoyo, Tokyo, Japan), to obtain the established dimensions [29].

The samples that received chemical polishing were submitted to a recoating of the photopolymerized glaze (Megadenta; Radeberg, Germany), with a fine, uniform layer being applied on the surface using a soft brush in one direction to avoid air bubbles. After a period of $20 \mathrm{~s}$, photopolymerization was performed for $180 \mathrm{~s}$ (Strobolux; EDG Equipamentos, Sao Carlos, Sao Paulo, Brazil), according to the manufacturer's recommendations. Prior to the tests, the samples were stored in an artificial saliva solution in a digital bacterial incubator (CIENLAB Equipamentos Cientificos Ltda, Campinas, Sao Paulo, Brazil) at $37 \pm 1{ }^{\circ} \mathrm{C}$ for $24 \mathrm{~h}$ [30].

Thereafter, tests for color alteration and microhardness were performed. For the surface characterization, atomic force microscopy (AFM), scanning electron microscopy (SEM), and energy dispersive $\mathrm{x}$ ray spectrometry (EDS) were performed. The same tests were performed again after 2000 thermocycles (t1) and after 21 days of sample immersion in coffee staining solution $(\mathrm{t} 2)$.

\subsection{Color analysis}

The color alterations were calculated by means of the CIE L*a*b* system, in accordance with the Comissim Internacionale de I'Eclairage CIE (International Commission of Illumination) norms [31].

\subsection{Microhardness analysis}

The surface microhardness was analyzed by means of the HMV-2 T microdurometer (Shimadzu Corp, Kyoto, Japan), following ASTM (American Society for Testing Materials) specifications [32].

\subsection{Atomic force microscopy}

For the AFM analysis, an additional sample from each group was fabricated for each test period. The images produced were transferred to the Gwyddion 2.33 software program (Nanometrology Department, Czech Institute of Nanometrology, Prague, Czech Republic) to obtain 3-D images $(5 \times 5 \mu \mathrm{m})$.

\subsection{SEM-EDS}

The SEM analysis (JSM 610LA; JEOL, Tokyo, Japan) was performed on an additional sample of each group for each test period, with the registered images magnified $300 \times$. The EDS analysis was performed simultaneously with the SEM on the order of $1 \mu \mathrm{m}^{3}$ [33].

\subsection{Thermocycling}

All samples were thermocycled (Convel, Sao Paulo, Sao Paulo, Brazil) by being immersed in distilled water and performing alternated baths of $30 \mathrm{~s}$ at a temperature of $5 \pm 1{ }^{\circ} \mathrm{C}$ and $55 \pm 1{ }^{\circ} \mathrm{C}$, totaling 2000 cycles [34].

\subsection{Immersion in coffee staining solution}

During the immersion process in coffee staining solution (Cafe Pilao; Sara Lee, Jundiai, Sao Paulo, Brazil), each sample was placed in a 
recipient filled with $1 \mathrm{~mL}$ of solution, prepared previously according to the manufacturer's recommendations, and the recipient was sealed to prevent evaporation of the solution. The samples remained stored in an incubator at $37 \pm 1{ }^{\circ} \mathrm{C}$ for $4 \mathrm{~h}$ per day for 21 days. While not in the coffee solution, the samples were stored in artificial saliva [35]. The 24-h in vitro storage simulated the consumption of coffee for 1 month [36]. After the immersion period, all samples were washed in running water for $1 \mathrm{~min}$, with the excess water being removed with paper absorbents [37]. Subsequently, new readings were performed in the same manner used prior.

\subsection{Statistical analysis}

The color analysis and surface microhardness quantitative data were submitted to the 1-way repeated-measures analysis of variance (ANOVA), followed by an appropriate test to compare the measurements among the groups (Tukey or Student $t$-test) with a 5\% significance $(P<0.05)$. The qualitative data of the AFM, SEM, and EDS were compared visually.

\section{Results}

In relation to the color alteration analysis, material $(P=0.014)$, treatment $(P=0.039)$, and period $(P=0.007)$ affected the results, and also the interaction between the material tested and the treatment $(P<0.001)$ (Table 1$)$.

All tested materials suffered color alteration since they presented $\Delta \mathrm{E}$ values $>0$ (Table 2 ). The CAAR resin had the greatest color alteration, statistically different from the Structur resin, which presented the least alteration. The Protemp resin presented greater values numerically, compared with the Charisma and Structur resins. No statistically significant difference occurred among the acrylic resin (HPAR and CAAR) values. However, the CAAR presented greater values numerically (Table 2).

Means followed by same uppercase letter in column do not differ from 5\% significance level $(P<0.05)$ for Tukey test.

After thermocycling, the color alteration of the samples was statistically greater than after the immersion period in the coffee solution (Table 3 ).

Table 1

3-Way Variance Analysis (ANOVA) with repeated measurements for color alteration of restorative materials used.

\begin{tabular}{llllll}
\hline Variation factors & $d f$ & SS & MS & F & $P$ \\
\hline Material & 4 & 132.944 & 33.236 & 3.299 & $0.014^{*}$ \\
Treatment (glaze) & 1 & 43.992 & 43.992 & 4.366 & $0.039^{*}$ \\
Material x Treatment & 4 & 490.818 & 122.704 & 12.178 & $<0.001^{*}$ \\
Between samples & 90 & 906.815 & 10.076 & & \\
Period & 1 & 43.955 & 43.955 & 7.685 & $0.007^{*}$ \\
Period x Material & 4 & 52.376 & 13.094 & 2.289 & 0.066 \\
Period x Treatment & 1 & 2.298 & 2.298 & 0.402 & 0.528 \\
Material x Period x Treatment & 4 & 55.741 & 13.935 & 2.436 & 0.053 \\
Intra samples & 90 & 514.779 & 5.720 & & \\
\hline
\end{tabular}

* $P<0.05$ denotes statistically significant difference.

Table 2

Mean values (standard deviation) of color alteration $(\Delta E)$ of each restorative material used, independent of period and treatment (glaze).

\begin{tabular}{ll}
\hline Material & $\Delta \mathrm{E}$ \\
\hline Protemp & $7.27(3.00) \mathrm{AB}$ \\
Charisma & $5.95(1.95) \mathrm{AB}$ \\
CAAR & $7.80(2.98) \mathrm{A}$ \\
HPAR & $7.20(3.70) \mathrm{AB}$ \\
Structur & $5.81(2.24) \mathrm{B}$ \\
\hline
\end{tabular}

Means followed by same uppercase letter in column do not differ from $5 \%$ significance level $(\mathrm{P}<0.05)$ for Tukey test.
Table 3

Mean values (standard deviation) of color alteration $(\Delta \mathrm{E})$ of material after thermocycling ( $\mathrm{t} 1)$ and after immersion (t2), independent of restoration material and treatment (glaze).

\begin{tabular}{ll}
\hline Periods & $\Delta \mathrm{E}$ \\
\hline $\mathrm{t} 1$ & $7.28(3.70) \mathrm{A}$ \\
$\mathrm{t} 2$ & $6.34(2.91) \mathrm{B}$ \\
\hline
\end{tabular}

Means followed by same uppercase letter in column do not differ from $5 \%$ significance level $(\mathrm{P}<0.05)$ for Student t-test

\section{Table 4}

Mean values (standard deviation) of color alteration $(\Delta E)$ of material for each treatment (glaze) used, independent of restoration material and period.

\begin{tabular}{ll}
\hline Treatment & $\Delta \mathrm{E}$ \\
\hline With glaze & $6.34(3.10) \mathrm{B}$ \\
Without glaze & $7.28(3.54) \mathrm{A}$ \\
\hline
\end{tabular}

Means followed by same uppercase letter in column do not differ from 5\% significance level $(\mathrm{P}<0.05)$ for Student t-test.

Analyzing treatment types, the samples that did not receive a photopolymerized glaze application presented a greater color alteration than the samples with glaze, with a statistical difference (Table 4). It can be observed that treatment with glaze significantly influenced the color alteration, increasing the $\Delta \mathrm{E}$ values of samples fabricated with CAAR and decreasing the $\Delta \mathrm{E}$ values of samples fabricated with HPAR (Table 5).

Table 5

Mean values (standard deviation) of color alteration $(\Delta E)$ of each restorative material with or without glaze, independent of period.

\begin{tabular}{ll}
\hline Material & $\Delta \mathrm{E}$ \\
\hline $\mathrm{A}$ & $7.44(2.63) \mathrm{ABC}$ \\
$\mathrm{B}$ & $7.18(3.36) \mathrm{BC}$ \\
$\mathrm{C}$ & $5.92(2.23) \mathrm{BCD}$ \\
$\mathrm{D}$ & $5.84(1.74) \mathrm{CD}$ \\
E & $6.54(2.14) \mathrm{B}$ \\
F & $9.16(3.07) \mathrm{AB}$ \\
$\mathrm{G}$ & $10.59(5.29) \mathrm{A}$ \\
$\mathrm{H}$ & $3.77(2.04) \mathrm{D}$ \\
I & $5.90(2.19) \mathrm{CD}$ \\
J & $5.74(2.33) \mathrm{CD}$ \\
\hline
\end{tabular}

Means followed by same lowercase letter in column do not differ from $5 \%$ significance level $(P<0.05)$ for Tukey test. Protemp without glaze (A), Protemp with glaze (B), Charisma without glaze (C), Charisma with glaze (D), CAAR without glaze (E), CAAR with glaze (F), HPAR without glaze (G), HPAR with glaze $(H)$, Structur without glaze (I), Structur with glaze $(\mathrm{J})$.

Table 6

3-way variance analysis (ANOVA) of microhardness of restorative materials used.*

\begin{tabular}{llllll}
\hline Variation factors & $d f$ & SS & MS & F & $P$ \\
\hline Material & 4 & $44,325.429$ & $11,081.372$ & 1844.785 & $<0.001^{*}$ \\
Treatment (glaze) & 1 & 3647.053 & 3647.053 & 607.148 & $<0.001^{*}$ \\
Material x Treatment & 4 & $22,730.326$ & 5682.582 & 946.015 & $<0.001^{*}$ \\
Between samples & 90 & 540.618 & 6.007 & & \\
Period & 1.89 & 778.517 & 412.708 & 32.989 & $<0.001^{*}$ \\
Period x Material & 7.545 & 157.488 & 20.872 & 1.668 & 0.114 \\
Period x Treatment & 1.89 & 738.813 & 391.660 & 31.307 & $<0.001^{*}$ \\
Material x Period x & 7.545 & 301.362 & 39.940 & 3.192 & $0.003^{*}$ \\
$\quad$ Treatment & & & & & \\
Intra samples & 180 & 2123.939 & 11.800 & & \\
\hline
\end{tabular}

$* P<0.05$ denotes statistically significant difference. 


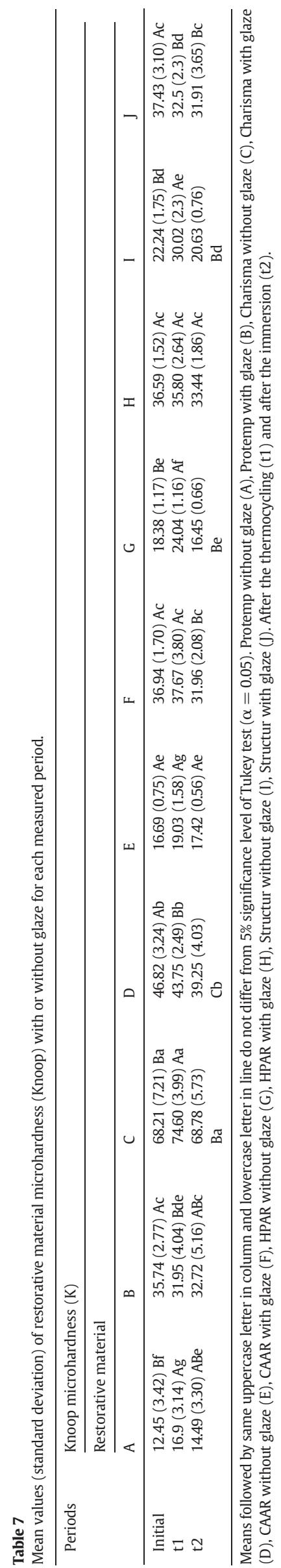

Means followed by same uppercase letter in column do not differ from 5\% significance level $(P<0.05)$ for Student $t$-test.

Means followed by same uppercase letter in column do not differ from $5 \%$ significance level $(P<0.05)$ for Student $t$-test.

Means followed by same lowercase letter in column do not differ from 5\% significance level $(P<0.05)$ for Tukey test. Protemp without glaze (A), Protemp with glaze (B), Charisma without glaze (C), Charisma with glaze (D), CAAR without glaze (E), CAAR with glaze (F), HPAR without glaze (G), HPAR with glaze $(H)$, Structur without glaze (I), Structur with glaze (J).

Assessing the surface microhardness of the samples, an interaction among three factors (material, period, and treatment) affected the results (Table $6, P=0.003$ ).

Among all evaluated materials, Charisma composite resin presented the greatest statistically significant microhardness values in the initial periods after thermocycling and after immersion. The lowest microhardness values were verified for the Protemp resin without glaze (Table 7).

In general, a statistically significant increase in microhardness values after thermocycling for the groups without glaze was observed, and a decrease in values for groups with glaze (Table 7). A numerical decrease in microhardness values for all samples immersed in coffee staining solution was observed, with the exception of the Protemp group with glaze (Table 7).

In relation to the surface treatment, the application of glaze resulted in a statistically significant increase of microhardness levels of the samples, with the exception of the Charisma group which presented statistically smaller values (Table 7).

Means followed by same uppercase letter in column and lowercase letter in line do not differ from 5\% significance level of Tukey test ( $\alpha=$ 0.05). Protemp without glaze (A), Protemp with glaze (B), Charisma without glaze (C), Charisma with glaze (D), CAAR without glaze (E), CAAR with glaze (F), HPAR without glaze (G), HPAR with glaze $(H)$, Structur without glaze (I), Structur with glaze (J). After the thermocycling (t1) and after the immersion (t2).

The atomic force microscopy (AFM) images illustrate the surfaces of the samples in 3-dimensions. The visual analysis revealed the formation of the greatest irregularities, such as peaks and valleys, in the groups without the application of glaze. In the groups with glaze, regions without irregularities were seen in the initial period, and smaller irregularities formed after the thermocycling and immersion in coffee solution (Fig. 1).

By way of the SEM images with a $300 \times$ increase, greater porosity and degradation of the photopolymerized glaze can be seen on the surface of the Protemp resin samples after the thermocycling and immersion in coffee (Figs. 3 and 4, Group B). In the Structur group with glaze, independent of the period analyzed, porosities can be seen on the surfaces (Figs. 2 to 4 , Group I). However, compared with the other groups, no significant changes were verified on the surface of the samples compared with the initial period. In relation to the chemical composition of the surfaces using the EDS spectrum, principally carbon (C), oxygen (O), and palladium $(\mathrm{Pd})$ were detected in the groups submitted to mechanical polishing in the initial period. After the thermocycling and immersion in coffee solution, only the first two elements were detected. In the groups with chemical polishing, greater quantities of carbon (C) and oxygen (O) were detected in all periods (Figs. 2 to 4 ).

\section{Discussion}

The null hypothesis tested was not accepted since the application of glaze and the aging of the samples influenced the physical and mechanical properties of the analyzed materials.

The present study verified that all of the tested materials presented color alteration ( $\Delta \mathrm{E}>0$; Table 2 ), with the values encountered being clinically unacceptable $(\Delta \mathrm{E}>3.3)$ [17]. The alteration could be caused by intrinsic and extrinsic factors. Extrinsic factors are related to thermal changes, humidity, as well as absorption and adsorption of pigments [13]. 
Initial

A

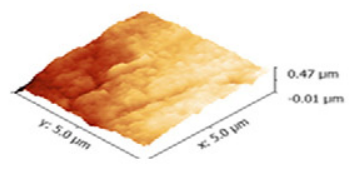

B

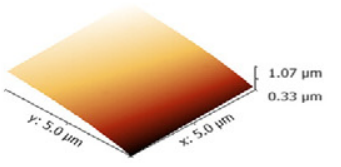

C

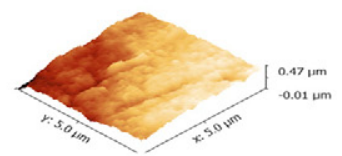

D

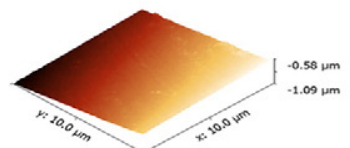

$\mathrm{E}$

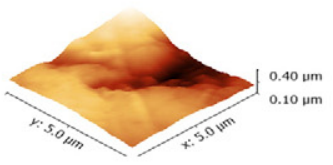

F

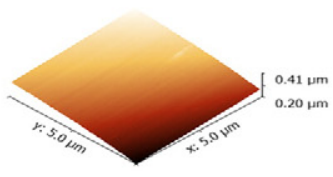

G

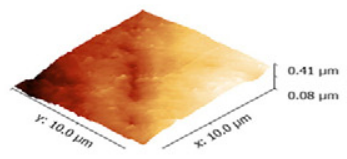

H

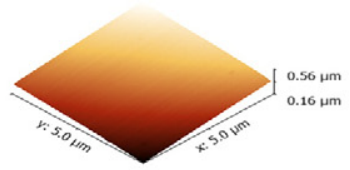

I

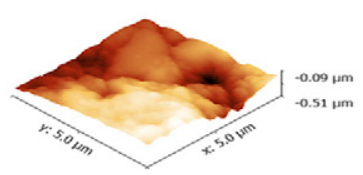

$\mathbf{J}$

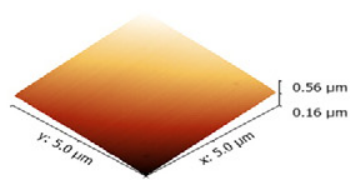

t1
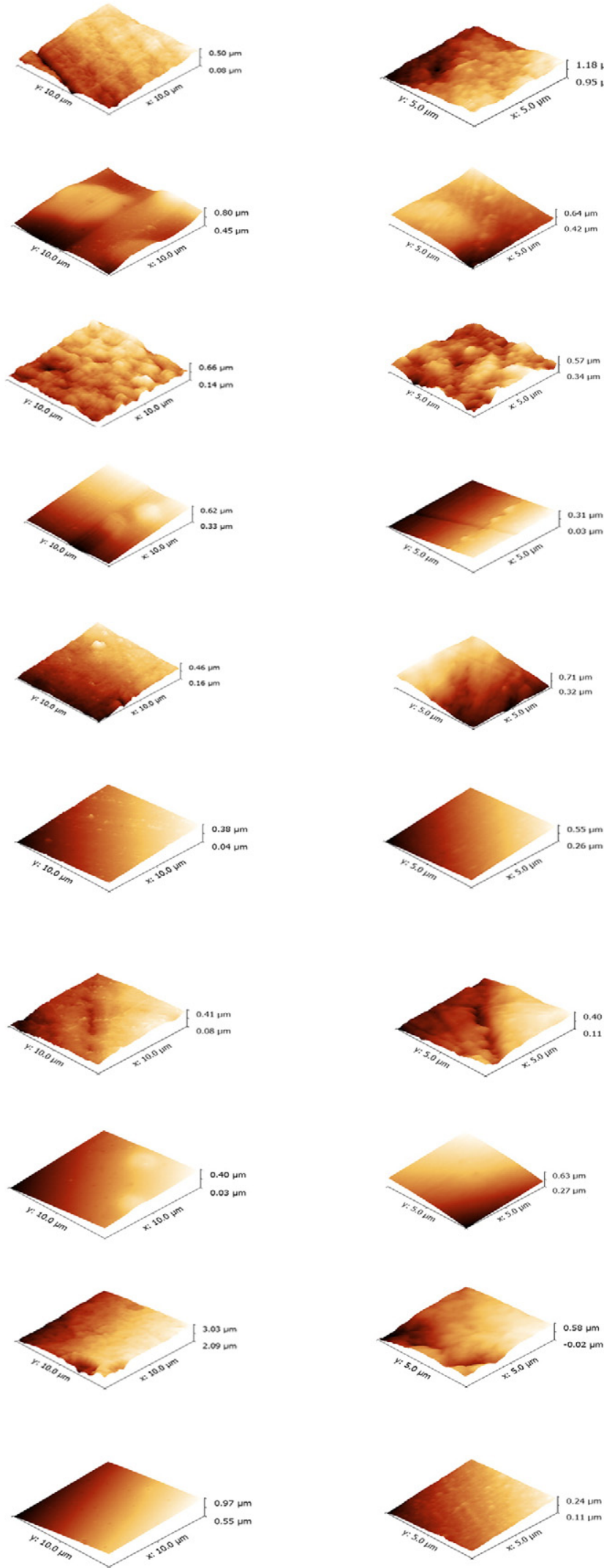

t2
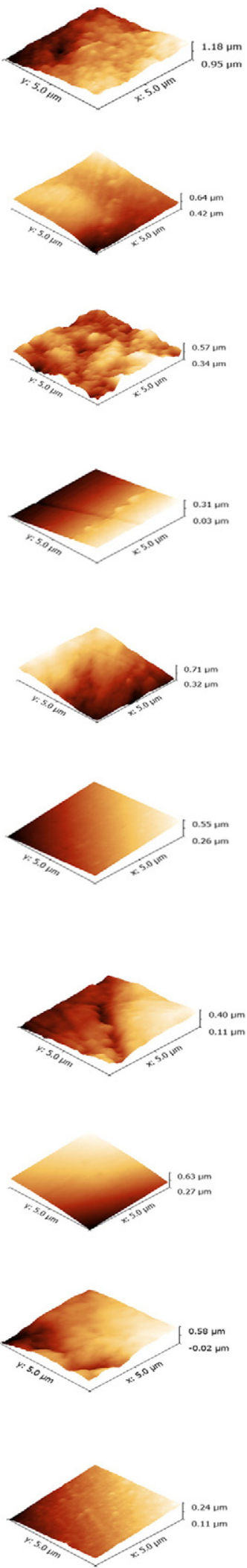

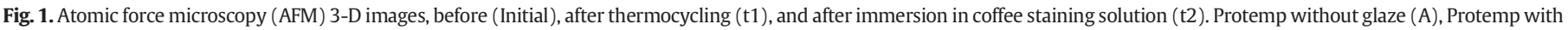

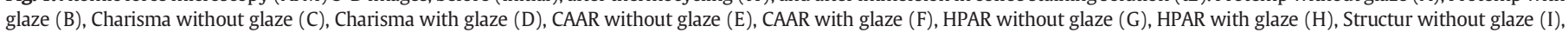
Structur with glaze (J). 

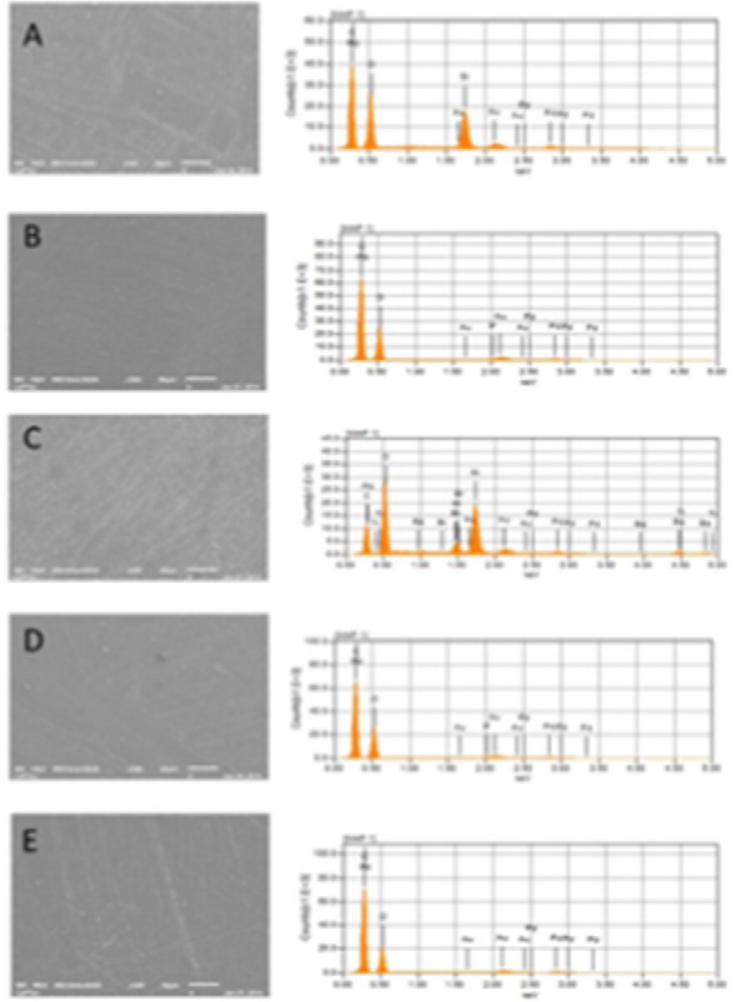
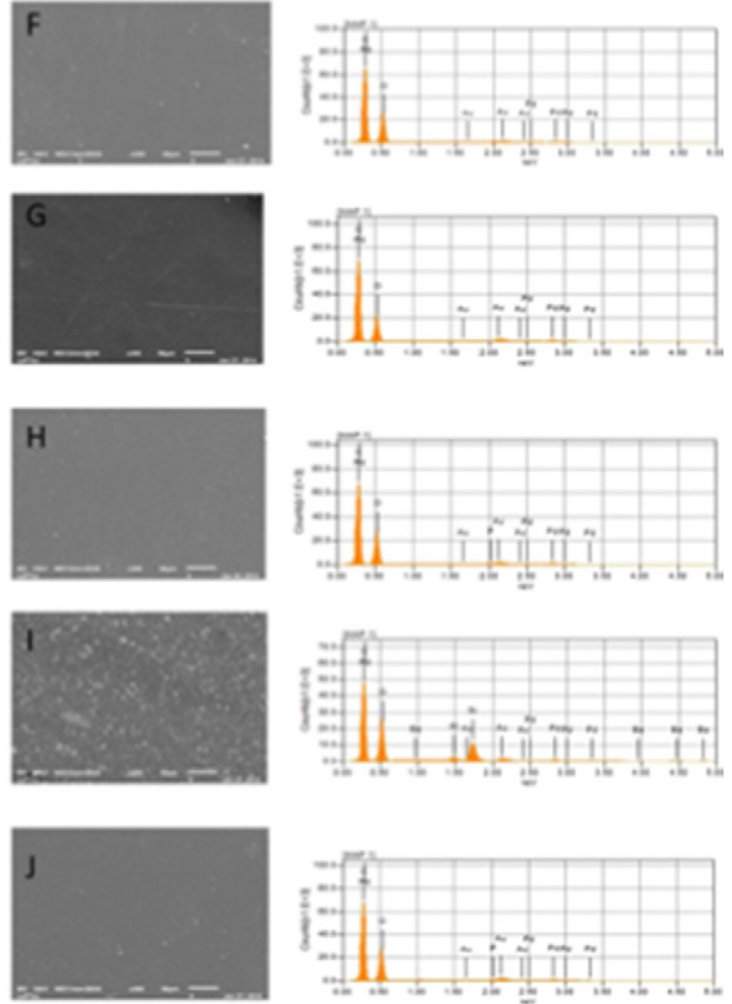

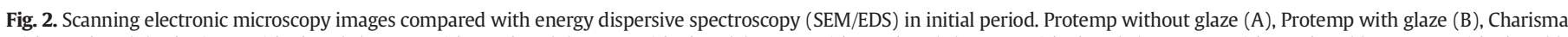

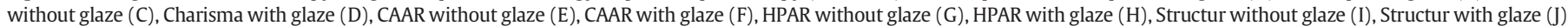
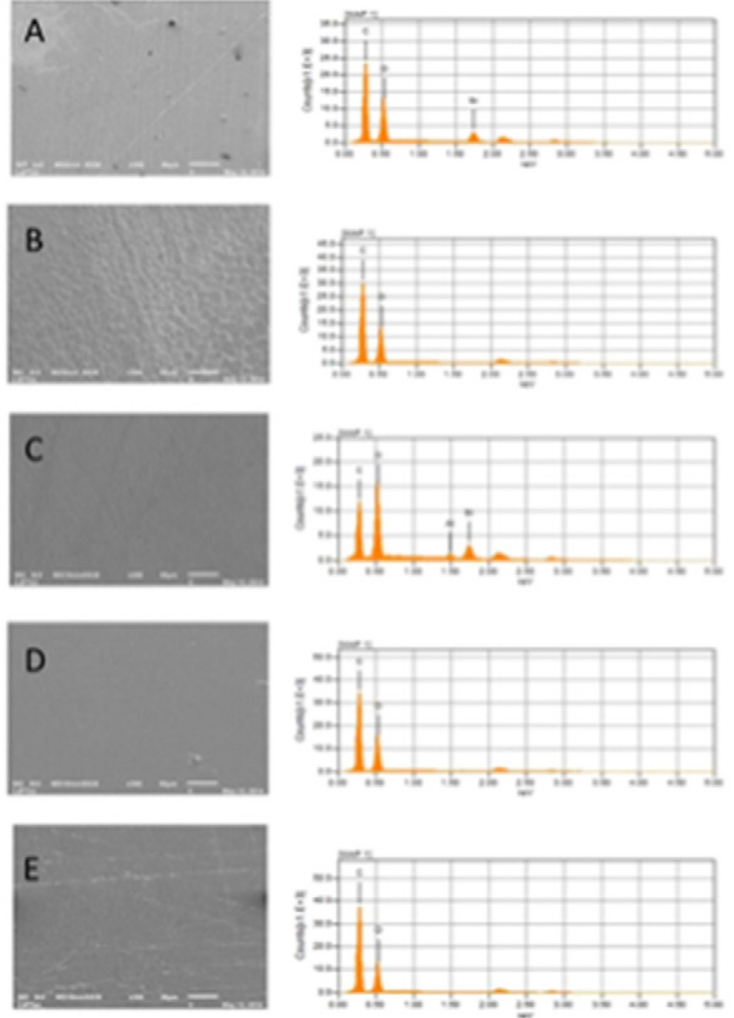
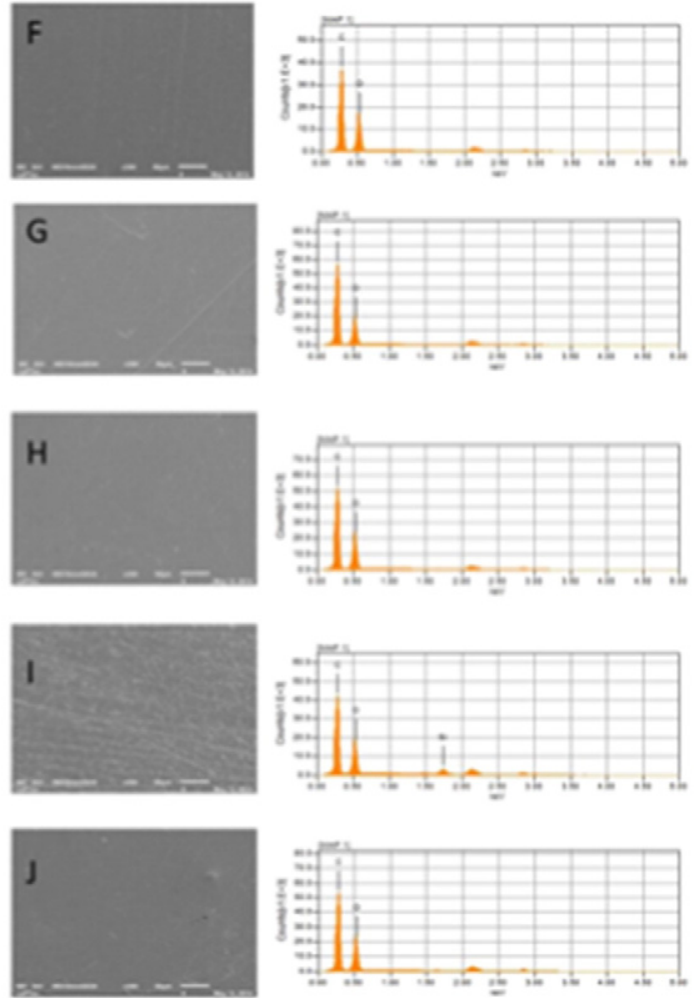

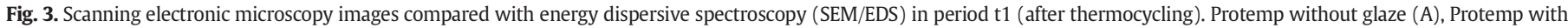

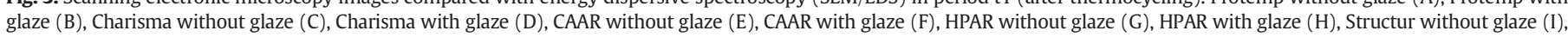
Structur with glaze $(\mathrm{J})$. 

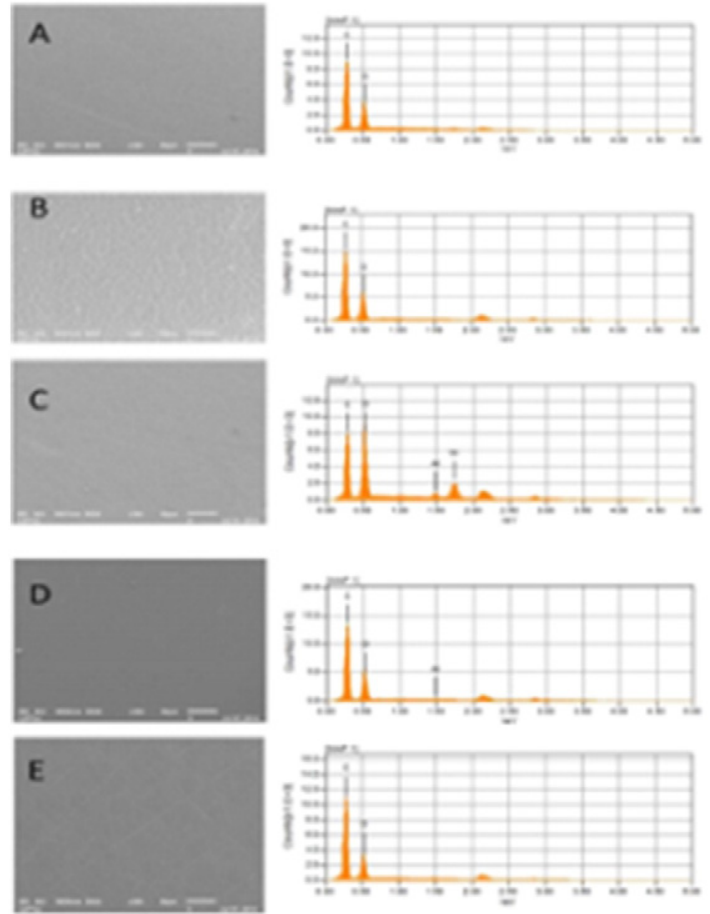
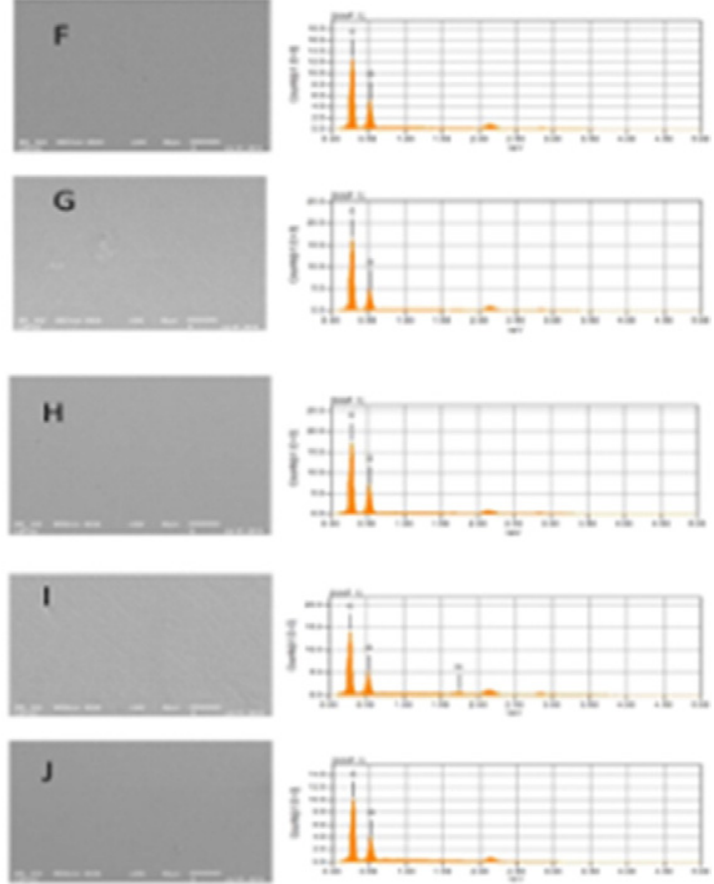

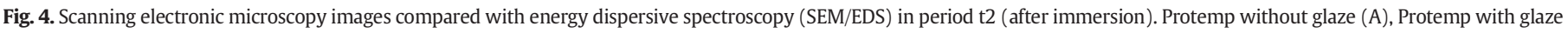

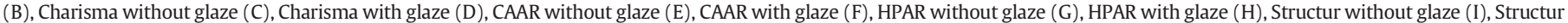
with glaze $(\mathrm{J})$.

The CAAR group presented the greatest values of color alteration, statistically significant compared with the Structur group (Table 2). This could have occurred due to the presence of the initiator monomer, benzoyl peroxide, in the polymer of the resin, and the activator agent, dimethyl p-toluidine, present in the monomer of the resin. These composites, in conjunction with the polymerization, promote surface yellowing of the CAAR and more severe chromatic alterations with the passing of time [31].

Resin composites (Bis-GMA and Bis-acryl) possess a heterogeneous chemical composition [5] which consists of an organic and inorganic matrix, and a bonding agent. The quantity of charged particles influences the water sorption process. This is related to the chromatic alteration of the polymers [1], which could have contributed to the smaller values of color alteration of the Charisma and Structur resins. In addition, according to the manufacturer, Charisma resin is composed of barium glass particles that have an average size of $0.7 \mu \mathrm{m}$ and a maximum size lower than $2 \mu \mathrm{m}$, forming an amorphous and compact structure that gives the material excellent optic properties.

The Protemp resin presented numerically greater color alteration values, compared with the Charisma resin, and greater statistically significant values compared with the Structur resin (Table 2); this is an interesting fact, since Protemp and Structur resins are bisacrylic resins and should present similar behaviors. However, the quantity of charged particles in bisacrylic resin can vary according to each manufacturer [4], influencing the mechanical properties of the material, as mentioned before.

The chromatic alteration of the CAAR showed itself to be numerically greater than that of the HPAR (Table 2). This occurred because autopolymerized resins present a high quantity of additional agents, such as benzoyl peroxide [16], which remain after the polymerization of the resin and could alternate the color of the material. Consider also that autopolymerizable resins present low rates of conversion during polymerization, producing a large quantity of monomer residual as a final product. This residual could interact with the pigments present in the polymer, deteriorating the color even further according to Bonatti et al. (2009), which affirms the degree of conversion and the presence of monomer residual as factors that could contribute to the chromatic alteration [14]. It must be emphasized that polymerization, water sorption, and consequently, chromatic stability, are factors that are influenced by resin chemicals, disposition, volume of particles, effectiveness of the initiator system, and the level of monomer polarity [2].

There was a greater color alteration when the samples were submitted to thermocycling (Table 3 ). This could be explained by the process of absorption and adsorption of water which the resins undergo, and which occurs until they are saturated [6]. Therefore, when the readings were performed after the thermocycling, the material was already saturated. When immersed in the coffee solution, the staining molecules are not able to penetrate the resinous matrix of the material [1], since it is already chemically stable and, consequently, the decrease in $\Delta \mathrm{E}$ values occurred.

The use of photopolymerized glaze decreased the color alteration values (Table 4). According to Rutkunas et al. (2010), the treatment with glaze increases the resistance to coloration. It is believed that this fact could have occurred due to the formation of a surface layer produced by the glaze, which protects the resin from the color pigments since the polymer surfaces remain exposed in the samples without glaze, causing an increase in the $\Delta \mathrm{E}$ values [1].

It can also be observed that the groups that were submitted to glaze presented the smaller color values, with the exception of the CAAR (Table 5), which showed a statistically significant difference between its values with and without glaze. It is believed that this occurred due to the glaze interacting with its residual monomers, leading to its degradation and consequent resin color alteration. In relation to the composite resins (Bis-GMA and Bis-acryl), a decrease of color alteration values was not verified among the treatments with or without glaze (Table 5).

In relation to the microhardness test, it can be observed that the Charisma composite resin presented the greatest values with statistically 
significant difference compared with the other resins (Table 7). This could have occurred due to the different chemical compositions of each material. Charisma resin is composed of a greater number of inorganic particles compared with the other resins tested, which increases its microhardness [7].

The Protemp and Structur bisacrylic resins presented a difference among the microhardness values (Table 7). This fact is related to the different chemical compositions among them, principally in relation to the quantity of inorganic particles, or even by the quantity of bifunctional monomers capable of realizing a greater number of cross-links [4].

The microhardness values of the HPAR and CAAR resins without glaze were not statistically different. However, numerically, it can be verified that the CAAR had smaller values in the initial period (Table 7). This could have occurred due to the presence of monomer residual present in greater quantities in the CAAR. This monomer possesses a plasticizing action, increasing the water sorption and, consequently, the resin solubility, which reduces the microhardness values [3].

The Protemp resin without glaze presented the smallest microhardness values in all of the analyzed periods, statistically different when compared with the values of the other resins. This fact was interesting, since a result similar to the Structur resin was expected (Table 7). Beyond the fact that the materials presented different chemical compositions, this result can be explained due to bisacrylic resins presenting a high degree of reticulation, which makes them less resistant, reflecting directly in smaller microhardness values [36].

The thermocycling process statistically increases the microhardness values of the samples for the groups without glaze (Table 7). Some authors suggest that the $55{ }^{\circ} \mathrm{C}$ water temperature during the thermocycling process contributed to the material undergoing an extra polymerization, making it more resistant [34]. The microhardness values for the samples submitted to the treatment with glaze after thermocycling were smaller, which could have resulted in degradation of the glaze surface.

The immersion of the samples in coffee solution numerically decreased the microhardness values, except for the Protemp group with glaze (Table 7). This can be attributed to the degree of water absorption and hydrophilicity of the resinous matrix of each material evaluated, and could lead to hydrolytic degradation among the particles and organic matrix, leading to alterations in the physical and mechanical properties of the materials [37]. This hydrolysis appears to have also provoked a detachment of the glaze layer. The AFM (Figs. 1B, D, F, H, J) and SEM (Figs. 3B, D, F, H, J e 4B, 4D, 4F, 4H, 4 J) images illustrate this fact well, in which a smaller degradation, and surface porosities of the samples, can be observed.

With the exception of the Charisma group, the surface treatment with glaze increased the microhardness values (Table 7). The glaze applied over the surface of polymers could be capable of decreasing the leaching of the residual monomer, or even impede the absorption of water [3] which could have interfered with the microhardness values. In addition, it is assumed that the surface layer of photopolymerized glaze presents greater microhardness levels in relation to the HPAR, CAAR, Protemp, and Structur resin values; as the microdurometer (Knoop) measuring tip reads only the surface of the samples, it is probable that the reading will be performed only on the surface of the glaze, and not on the resins. This also occurred in the Charisma group. However, the microhardness values decreased with the application of the glaze, demonstrating that the Charisma resin presents a greater microhardness value than the layer of glaze on which the reading was performed.

This study presents some limitations since there are few studies to compare it to with the results obtained. It is evident that future studies are needed to evaluate and compare the durability, chemical stability, and resistance in adverse conditions, such as cleaning processes, with the purpose of identifying surface treatments that optimize the properties of the materials.

\section{Conclusions}

Due to the results obtained, and considering the limitations of this study, it can be concluded, in relation to the color, that the groups with glaze present smaller color alteration. In addition, Charisma and Structur resins exhibited the greatest chromatic stability. As to the microhardness, the values were greater when the samples were treated with the glaze, with the exception of the Charisma group.

\section{Acknowledgments}

The authors thank Elton José de Souza for the Atomic Force Microscopy Analysis performed at the Faculty of Engineering of Ilha Solteira FEIS/UNESP, Ilha Solteira, Sao Paulo, Brazil. Additionally, the authors thank FAPESP (Foundation for Support to Research of the State of Sao Paulo) for financial support [n. 2013/21383-5] provided to Betina Chiarelo Commar.

\section{References}

[1] V. Rutkunas, V. Sabaliauskas, H. Mizutani, Effects of different food colorants and polishing techniques on color stability of provisional prosthetic materials, Dent. Mater. J. 29 (2010) 167-176.

[2] D.R. Haselton, A.M. Diaz-Arnold, D.V. Dawson, Color stability of provisional crown and fixed partial denture resins, J. Prosthet. Dent. 93 (2005) 70-75.

[3] G.A. Thompson, Q. Luo, Contribution of postpolymerization conditioning and storage environments to the mechanical properties of three interim restorative materials, J. Prosthet. Dent. 112 (2014) 638-648.

[4] D.R. Haselton, A.M. Diaz-Arnold, M.A. Vargas, Flexural strength of provisional crown and fixed partial denture resins, J. Prosthet. Dent. 87 (2002) 225-228.

[5] S. Turgut, B. Bagis, E.A. Ayaz, K.U. Ulusoy, S.H. Altintas, F.M. Korkmaz, et al., Discoloration of provisional restorations after oral rinses, Int. J. Med. Sci. 10 (2013) 1503-1509.

[6] T.N. Rahim, D. Mohamad, H. Md Akil, I. Ab Rahman, Water sorption characteristics of restorative dental composites immersed in acidic drinks, Dent. Mater. 28 (2012) e63-e70.

[7] W.W. Johnson, V.B. Dhuru, W.A. Brantley, Composite microfiller content and its effect on fracture toughness and diametral tensile strength, Dent. Mater. 9 (1993) 95-98.

[8] E.J.J.R. Givens, G. Neiva, P. Yaman, J.B. Dennison, Marginal adaptation and color stability of four provisional materials, J. Prosthodont 17 (2008) 97-101.

[9] J.L. Ferracane, Hygroscopic and hydrolytic effects in dental polymer networks, Dent. Mater. 22 (2006) 211-222.

[10] D.M. dos Santos, B.E. Nagay, E.V.F. da Silva, L.R. Bonatto, M.V. Sonego, et al., In vitro analysis of different properties of acrylic resins for ocular prosthesis submitted to accelerated aging with or without photopolymerized glaze, Mater. Sci. Eng. C Mater. Biol. Appl. 69 (2016) 995-1003.

[11] G. Migliau, L.K. Besharat, A.A. Sofan, E.A. Sofan, U. Romeo, Endo-restorative treatment of a severly discolored upper incisor: resolution of the "aesthetic" problem through Componeer veneering system, Ann. Stomatol. (Roma) 6 (2016) 113-118

[12] S. Turgut, B. Bagis, Colour stability of laminate veneers: an in vitro study, J. Dent. 39 (2011) e57-e64.

[13] N. Abu-Bakr, L. Han, A. Okamoto, M. Iwaru, Color stability of compomer after immersion in various media, J. Esthet. Dent. 12 (2000) 258-263.

[14] M.R. Bonatti, T.R. Cunha, R.R. Regis, C.H. Silva-Lovato, H.F. Paranhos, R.F. de Souza The effect of polymerization cycles on color stability of microwave processed denture base resin, J. Prosthodont. 18 (2009) 432-437.

[15] A.U. Fernandes, M.C. Goiato, D.M. dos Santos, Effect of weathering and thickness on the opacity of acrylic resin and ocular button for artificial eyes, J. Craniofac. Surg. 21 (2010) 64-67.

[16] M. Rosentritt, J. Esch, M. Behr, A. Leibrock, G. Handel, In vivo color stability of resin composite veneers and acrylic resin teeth in removable partial dentures, Quintessence Int. 29 (1998) 517-522.

[17] J.F. McCabe, Applied Dental Materials, seventh ed Blackwell Scientific Publications, Oxford, 1990 78-86.

[18] M.C. Goiato, D.M. dos Santos, H.G. Gennari- Filho, A.C. Zavanelli, S.F.C. Dekon, D.N. Mancuso, Influence of investment, disinfection, and storage on the microhardness of ocular resins, J. Prosthodont. 18 (2009) 32-35.

[19] K.A. Schulze, S.J. Marshall, S.A. Gansky, G.W. Marshall, Color stability and hardness in dental composites after accelerated aging, Dent. Mater. 19 (2003) 612-619.

[20] F. Aykent, I. Yondem, A.G. Ozyesil, S.K. Gunal, M.C. Avunduk, S. Ozkan, Effect of different finishing techniques for restorative materials on surface roughness and bacterial adhesion, J. Prosthet. Dent. 103 (2010) 221-227.

[21] D.R. Haselton, A.M. Diaz-Arnold, D.V. Dawson, Effect of storage solution solution on surface roughness of provisional crown and fixed partial denture materials, J. Prosthodont. 13 (2004) 227-232.

[22] D. Atabek, H. Sillelioglu, A. Olmez, The efficiency of a new polishing material: nanotechnology liquid polish, Oper. Dent. 35 (2010) 362-369. 
[23] D.R. Monteiro, L.F. Gorup, A.S. Takamiya, E.R. de Camargo, A.C. Filho, D.B. Barbosa, Silver distribution and release from an antimicrobial denture base resin containing silver colloidal nanoparticles, J. Prosthodont 21 (2012) 7-15.

24] A. Catelan, A.L. Briso, R.H. Sundfeld, M.C. Goiato, P.H. dos Santos, Color stability of sealed composite resin restorative materials after ultraviolet artificial aging and immersion staining solutions, J. Prosthet. Dent. 105 (2011) 236-241.

[25] J.C. de Oliveira, G. Aiello, B. Mendes, V.M. Urban, N.H. Campanha, J.H. Jorge, Effect of storage in water and thermocycling on hardness and roughness of resin materials for temporary restorations, Mat. Res. 13 (2010) 355-359.

[26] A. Moreno, M.C. Goiato, D.M. dos Santos, M.F. Haddad, A.A. Pesqueira, L.C. Bannwart, Effect of different disinfectants on the microhardness and roughness of acrylic resins for ocular prosthesis, Gerodontology 30 (2013) 32-39.

[27] M.C. Goiato, D.M. dos Santos, A. Moreno, M.G. Iyda, M.C. Rezende, M.F. Haddad, Effect of disinfection and storage on the flexural strength of ocular prosthetic acrylic resins, Gerodontology 29 (2012) e838-e844.

[28] M.C. Goiato, D.M. dos Santos, M.F. Haddad, A.A. Pesqueira, Effect of accelerated aging on the microhardness and color stability of flexible resins for dentures, Braz. Oral Res. 24 (2010) 114-119.

[29] A.U. Fernandes, M.C. Goiato, D.M. dos Santos, Effect of weathering and thickness on the superficial microhardness of acrylic resin and ocular button, Cont. Lens Anterior Eye 32 (2009) 283-287.

30] V. Colucci, C.D. dos Santos, F.L do Amaral, S.A. corona, A.B. Catirse, Influence of $\mathrm{NaHCO} 3$ powder on translucency of microfilled composite resin immersed in different mouthrinses, J. Esthet. Restor. Dent. 21 (2009) 242-248.

[31] K. Hoshiai, Y. Tanaka, K. Hiranuma, Comparison of a new autocuring temporary acrylic resin with existing products, J. Prosthet. Dent. 79 (1998) 273-277.

[32] ASTM E384 - 11e1 (Standard Test Method for Knoop and Vickers Hardness of Materials), 2012 available at http: enterprise.astm.org/filtrexx40.cgi?+REDLINE_PAGES/ E384.htm.

[33] D.M. dos Santos, E.V. da Silva, A.J. Vechiato-Filho, P.F. Cesar, E.C. Rangel, N.C. da Cruz, et al., Aging effect of atmospheric air on lithium disilicate ceramic after nonthermal plasma treatment, J. Prosthet. Dent. 115 (2016) 780-787.

[34] M.C. Goiato, B.C. Zuccolotti, D.M. dos Santos, M.A. Sinhoretti, A. Moreno, Effect of intrinsic nanoparticle pigmentation on the color stability of denture base acrylic resins, J. Prosthet. Dent. 110 (2013) 101-106.

35] R.O. Souza, M. Ozcan, S.M. Michida, R.M. de Melo, C.A. Pavanelli, M.A. Bottino, et at Conversion degree of indirect resin composites and effect of thermocycling on their physical properties, J. Prosthodont. 19 (2010) 218-225.

[36] R.E. Kerby, L.A. Knobloch, S. Sharples, A. Peregrina, Mechanical properties of urethane and bis-acryl interim resin materials, J. Prosthet. Dent. 110 (2013) 21-28.

[37] K.J. Söderholm, M. Zigan, M. Ragan, W. Fischlschweiger, M. Bergman, Hydrolytic degradation of dental composites, J. Dent. Res. 63 (1984) 1248-1254.

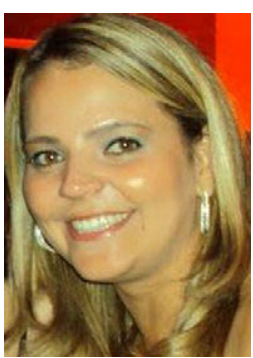

Daniela Micheline dos Santos received her PhD degree from the Aracatuba Dental School, Aracatuba Dental School, São Paulo State University (UNESP), Brazil. Currently, she is a teacher in the Department of Dental Materials and Prosthodontic at the same university.

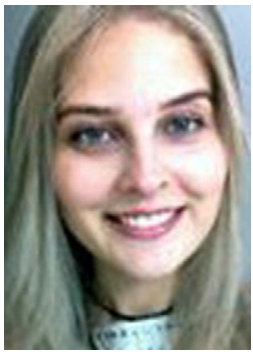

Betina Chiarelo Commar is currently a graduate student at the Aracatuba Dental School, São Paulo State University, Brazil.

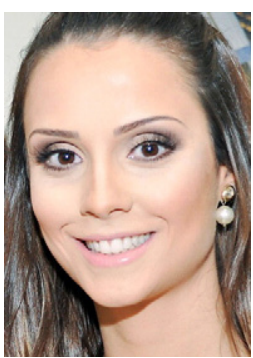

Liliane da Rocha Bonatto received her MS degree from the Aracatuba Dental School, São Paulo State University, Brazil. Currently, she is a teacher at the Paranaense University (UNIPAR).

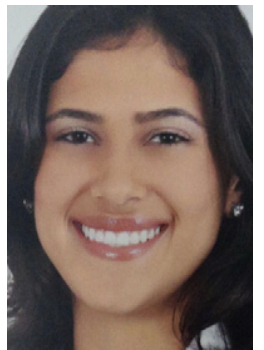

Emily Vivianne Freitas da Silva received her MS degree from the Aracatuba Dental School, Sao Paulo State University, Brazil. Currently, she is a postgraduate student at the same university.

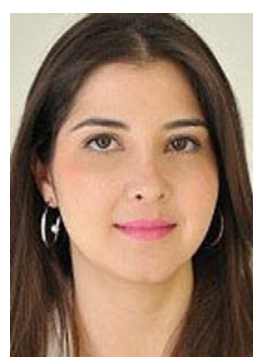

Mariana Vilela Sonego received her MS degree from the Aracatuba Dental School, São Paulo State University, Brazil. Currently, she is a postgraduate student at the same university.

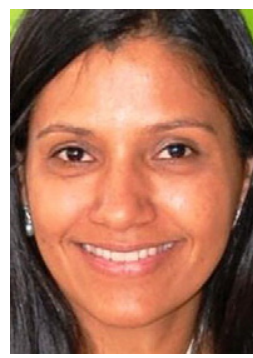

Elidiane Cipriano Rangel received her $\mathrm{PhD}$ degree from the Institute of Physics, State University of Campinas, Brazil. Currently, she is a teacher at the Institute of Science and Technology of Sorocaba, São Paulo State University, Brazil.

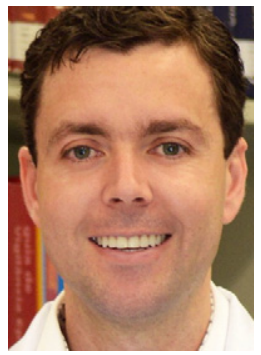

Aldieris Alves Pesqueira received her PhD degree from the Aracatuba Dental School, Aracatuba Dental School, Sao Paulo State University (UNESP), Brazil. Currently, she is a teacher in the Department of Dental Materials and Prosthodontics at the same university.

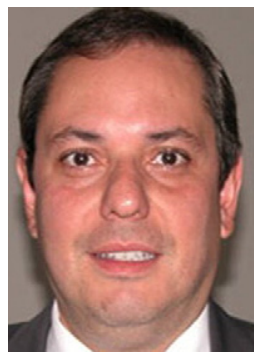

Marcelo Coelho Goiato received his $\mathrm{PhD}$ degree from the Dental School of Piracicaba, State University of Campinas, Brazil. Currently, he is a teacher in the Department of Dental Materials and Prosthodontics, Aracatuba Dental School, Sao Paulo State University, Brazil. 